Article

\title{
Novel Calibration of MIESM and Reduction of CQI Feedback for Improved Fast Link Adaptation
}

\author{
Hyunjee Lee ${ }^{1}$, Haseong Kim ${ }^{1}$ and Hosung Park ${ }^{2, *(\mathbb{D}}$ \\ 1 Department of LG Smart Hybrid Engineering, Chonnam National University, Gwangju 61186, Korea; \\ hglee0306@naver.com (H.L.); nivell@nate.com (H.K.) \\ 2 School of Electronics and Computer Engineering, Chonnam National University, Gwangju 61186, Korea \\ * Correspondence: hpark1@jnu.ac.kr; Tel.: +82-62-530-1791
}

Received: 8 December 2018; Accepted: 10 January 2019; Published: 2 March 2019

\begin{abstract}
In mobile communications systems, fast link adaptation (FLA) aims to achieve high system throughput of each user by accurately determining a channel quality indicator (CQI) for feedback, which predicts the next channel based on the current channel state information. In this paper, we propose an improved calibration method of mutual information effective signal-to noise-ratio mapping (MIESM) to determine an accurate CQI feedback value in FLA. Our proposed calibration method derives the optimal calibration factors by considering various channel environments and setting the effective interval of effective signal-to-noise ratio, which is a single value compressing the information of channel characteristics at a time. The simulation is performed in various signal-to-noise ratio (SNR) ranges to account for the actual environments, and the calibration factors are derived from the proposed calibration method. The results show that the CQI feedback value from the derived calibration factors are more accurate than the existing calibration factors. In addition, we discuss a study regarding the time-coherence-based CQI feedback bit reduction scheme. Assuming that each channel is correlated to the previous and subsequent channels, we propose a method to reduce the number of CQI feedback bits adapted to the corresponding SNR regime. Through the simulation, we compare the system throughputs of the proposed adaptive CQI feedback and the conventional CQI feedback scheme. As a result, the proposed CQI feedback has almost the same system throughput as the conventional CQI feedback scheme, but the average number of feedback bits is reduced, thereby improving the efficiency of the communication.
\end{abstract}

Keywords: fast link adaptation (FLA); channel quality indicator (CQI); calibration method; calibration factor; CQI feedback

\section{Introduction}

In mobile communications systems, the channel experienced by each user is apt to having frequency selectivity and time variation. In this situation, the user selects a channel quality indicator (CQI) by estimating an appropriate link quality for the fading channel through fast link adaptation (FLA) and sends the feedback. The base station (BS) selects a modulation and coding scheme (MCS) for transmission based on the CQI feedback from the user [1,2]. If the channel condition is determined to be good enough, the BS increases the modulation order and/or coding rate. In the opposite case, the BS decreases them. This FLA process with resource allocation increases the throughput of the system while maintaining the quality of service (QoS) between the user and the BS [3-7].

Although the FLA can use various metrics to predict link quality [8-10], in this paper, we use mutual information effective signal-to-noise-ratio mapping (MIESM) [11,12], which is a tool to obtain an accurate link-quality metric (LQM) to determine the CQI value for the instantaneous channel. The MIESM utilizes the instantaneous signal-to-noise ratios (SNRs) of subcarriers to compute the 
effective SNR, which is a constant value representing the whole-channel quality. In this process, a calibration factor is used to calculate the effective SNR in the MIESM procedure. The optimal calibration factor enables the most accurate block error rate (BLER) prediction for a given channel, allowing reliable CQI selection.

There were a few research works handling the calibration of MIESM. In Reference [11], the calibration factor of MIESM and its derivation were addressed first, but no detailed calibration procedure was given. In Reference [12], the standard method of calibration was proposed. In Reference [13], the average method was proposed to reduce the complexity during the calibration compared to the standard method. In Reference [14], an approximated version of MIESM was proposed for 256 quadrature amplitude modulation (QAM) in long-term evolution (LTE), but no calibration procedure was required. All the existing calibration methods of MIESM were performed using only limited channel environments without consideration of various channel conditions of mobile communications. This is not an optimal method considering FLA in actual situations.

Accurate CQI estimation of the FLA enables reliable communication with high throughput between the user and the BS [15-17]. The CQI reporting method of the user includes periodic reporting of CQI feedback at regular intervals and aperiodic reporting, which feeds back the CQI according to the request of the BS. There are three types of CQI feedback: wideband, higher layer-configured subband feedback, and UE selected subband CQI feedback [18]. The subband is defined by dividing the entire downlink bandwidth (wideband) by the subband size according to the number of resource blocks. One subband consists of consecutive physical resource blocks (PRBs), and it is the smallest unit for CQI feedback reporting. An efficient CQI feedback reporting reduces the overhead of uplink communication between the user and the BS. The CQI is reported differently depending on the channel state information (CSI) [19]. Differential CQI was adopted to reduce the amount of feedback bits in multi-input multi-output (MIMO) situations of the third generation partnership project (3GPP) evolved universal terrestrial radio access (E-UTRA) [18]. The differential CQI feedback first obtains the wideband CQI from subband CQIs, and then calculates the difference between each subband CQI and the wideband CQI. It is noted that the differential CQI feedback scheme does not utilize channel correlations over time.

In this paper, we propose an improved calibration method for MIESM by deriving the optimal calibration factor to improve the accuracy of CQI feedback in FLA. The improvement of the proposed method comes from two technical advances. Firstly, a wide range of additive white Gaussian noise (AWGN) SNR values are considered in the calibration procedure to reflect various channel conditions of users in reality. Secondly, the proposed method sets an effective interval of the effective SNR to screen unnecessary channels which do not help the calibration eventually. To verify the proposed method, we propose an efficient algorithm to check the accuracy of CQIs calculated from the derived calibration factors. As a result, it was confirmed via the system throughput simulation that the calibration factors derived from the proposed method provide more accurate CQI values than the conventional ones. In addition, under the assumption that there is some correlation in time channels, we also propose a CQI feedback bit reduction scheme which is applicable no matter which scheme is used, i.e., wideband CQI or subband CQI. Based on the time correlation between adjacent channels, the CQI difference of two consecutive channels is mostly bounded, which enables reducing the number of bits used for CQI feedback. Although a reduced number of feedback bits are used, it is shown via simulations that the decrease of the system throughput is negligible, which proves that the proposed CQI feedback bit reduction scheme is efficient and also robust in various channel conditions.

The remainder of the paper is organized as follows: Section 2 explains MIESM-based FLA and the average calibration method. Section 3 presents the improved calibration method to obtain optimal calibration factors. Section 4 introduces the CQI feedback bit reduction schemes. Results of the simulations and the analysis are shown in Section 5. Finally, this paper is concluded in Section 6. 


\section{Preliminaries}

This section describes the MIESM-based FLA mechanism, and introduces the existing calibration method.

\subsection{MIESM-Based Fast Link Adaptation}

A user obtains the channel coefficient information from the orthogonal frequency division multiplexing (OFDM) symbols, determines a CQI suitable for the current channel through the MIESM-based FLA, and reports it to the BS. The goal of the FLA is to increase the throughput of the system while maintaining the target BLER for various channel conditions. The MIESM-based FLA process is described below [20].

Firstly, the effective SNR ( $\left(\mathrm{SNR}_{\mathrm{eff}}\right)$, which is a constant representing the channel quality, is calculated from the instantaneous SNRs of the subcarriers based on the estimated values of the channel state information. The equation for this is given in Reference [11] as follows:

$$
\mathrm{SNR}_{\mathrm{eff}}=f_{m}{ }^{-1}\left(\frac{1}{P} \sum_{p=1}^{P} f_{m}\left(\frac{\mathrm{SNR}_{p}}{\beta}\right)\right)
$$

where $P$ indicates the number of subcarriers, $\mathrm{SNR}_{p}$ is the instantaneous SNR of the $p$-th subcarrier, and $\beta$ is a calibration factor to minimize the difference between the estimated effective BLER (BLER eff $_{\text {) }}$ and the real BLER for the current channel in the calibration procedure. The function $f_{m}(\cdot)$ is the bit-interleaved coded modulation (BICM) capacity curve which depends on the modulation order $m$. This capacity is equivalent to the mutual information between channel input and output, which is the reason this process is called MIESM [21].

Secondly, the calculated $\mathrm{SNR}_{\text {eff }}$ is mapped through the pre-calculated AWGN/BLER look-up table

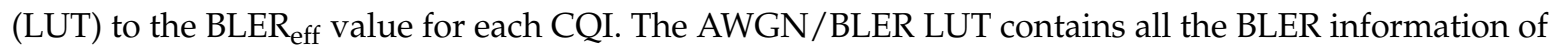
every CQI under the AWGN channel over various SNR values. Then, we select the largest CQI such

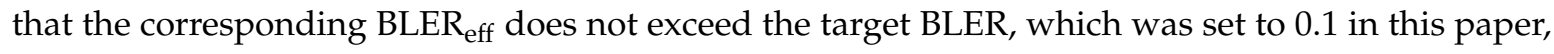
and feeds the selected CQI back to the BS.

\subsection{Average Method for Calibration of MIESM}

Accurate CQI determination is possible using appropriate calibration factors for effective SNR calculation. The standard calibration procedure was proposed in Reference [12], which requires a vast number of link-level simulations to include a significant number of channel instances. For this reason, in many cases, the optimal calibration factors are practically obtained from the average calibration procedure outlined in Reference [13].

Before describing the calibration procedure, we explain some notations. These notations are also used for the improved calibration method described in Section 3. $N_{b}$ is the number of candidate calibration factors for every CQI, and $\beta_{i, l}, l=1, \ldots, N_{b}$, is the $l$-th calibration factor for CQI $i . N_{s}$ is the length of AWGN SNR vectors to simulate in search of the optimal calibration factor, where the noise variance corresponding to each SNR is $\left[\sigma_{1}^{2}, \ldots, \sigma_{N_{s}}^{2}\right]$. The symbol $N_{c}$ is the number of channel realizations which should be big enough to show the characteristics of the entire channel, and $H_{k}$, $k=1, \ldots, N_{c}$ is the $k$-th channel instance. $N_{w}$ is the number of noise realizations and $w_{n}, n=1, \ldots, N_{w}$, is the $n$-th noise instance. Also, $\operatorname{BLER}_{\mathrm{LUT}}\left(\operatorname{SNR}_{\text {eff }}(\cdot, \cdot, \cdot)\right)$ is the effective BLER obtained by mapping the $\mathrm{SNR}_{\text {eff }}$ using the AWGN/BLER LUT.

The calibration steps in Algorithm 1 are further described below. $N_{c}$ channels and $N_{w}$ noises are generated for one calibration factor candidate and one AWGN SNR point. The instantaneous $\operatorname{BLER}\left(H_{k}, w_{n}\right)$ is calculated for the generated channel and noise by decoding. This value is 0 if the block is correct, or 1 otherwise. The average BLER $\left(\operatorname{BLER}\left(H_{k}, \sigma_{j}^{2}\right)\right)$ over noise is then calculated and accumulated. Then, the average $\operatorname{BLER}\left(\overline{\operatorname{BLER}}\left(\sigma_{j}^{2}\right)\right)$ over a given channel and noise realization 
is calculated and saved. The BLER $\mathrm{LUT}_{\text {is }}$ is estimated using $\mathrm{SNR}_{\text {eff }}$, and this is also averaged over the entire channel.

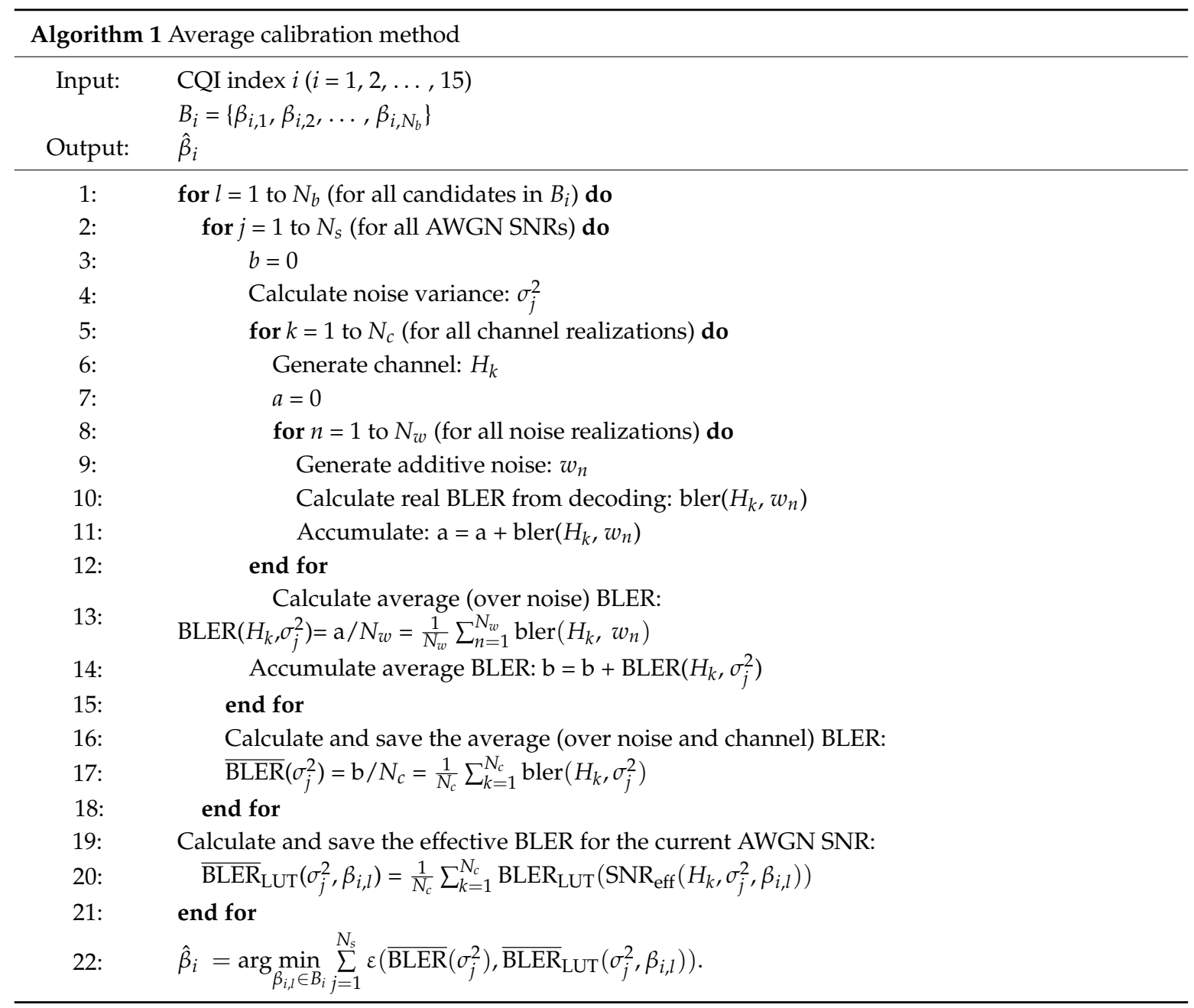

The purpose of the calibration is to predict the $\overline{\mathrm{BLER}}_{\mathrm{LUT}}$ as close as possible to the real average $\overline{\mathrm{BLER}}$ for all AWGN SNR values and channel conditions. For this, the above calibration procedure is necessary, and the optimal calibration factor is calculated as

$$
\beta_{o p t}^{a v}=\hat{\beta}_{i}=\arg \min _{\beta_{i, l} \in B_{i}} \sum_{j=1}^{N_{s}} \varepsilon\left(\overline{\operatorname{BLER}}\left(\sigma_{j}^{2}\right), \overline{\operatorname{BLER}}_{\mathrm{LUT}}\left(\sigma_{j}^{2}, \beta_{i, l}\right)\right),
$$

where $\varepsilon(x, y)$ is an error function that calculates the error by assigning equal weights to $x$ and $y$.

$$
\varepsilon(x, y)=\left|\log _{10}(x)-\log _{10}(y)\right|^{2} .
$$

In general, the AWGN SNR range of the average calibration procedure is limited to SNR points of AWGN/BLER 0.95 to BLER 0.01 for each CQI, since all data collected in the link-level simulation are not sufficiently reliable [13].

\section{Improved Calibration Method}

The existing calibration method has a limitation, whereby only the limited channel quality is considered because the calibration factor is derived by generating noise for the limited range of AWGN SNR corresponding to the BLER 0.95 to BLER 0.01 in order to find the optimum calibration factors. 
The purpose of the improved calibration method is to derive an optimal calibration factor for accurate CQI determination using various channels from a wide SNR range. The following two steps are required before applying the improved calibration method:

- The effective interval of the $\mathrm{SNR}_{\text {eff }}$ for correctness and efficiency of the calibration must be defined before deriving the calibration factor. Since the CQI feedback value is determined on the basis of the target BLER 0.1, the effective interval of $\mathrm{SNR}_{\text {eff }}$ is defined from the SNR value corresponding to AWGN BLER 0.9, which is denoted by $\gamma_{0.9}^{i}$, to the SNR value corresponding to AWGN BLER 0.001 , which is denoted by $\gamma_{0.001}^{i}$, for each CQI. The effective interval efficiently includes valid channels for the calibration around the SNR corresponding to AWGN BLER 0.1.

- The probability distribution of $\mathrm{SNR}_{\text {eff }}$ over a number of channels must be obtained for each given AWGN SNR and each CQI. The distribution of SNR eff for each AWGN SNR almost follows the Gaussian distribution as shown in Figure 1. Let $\mathrm{SNR}_{\text {eff }}^{s}$ denote the random variable corresponding to the effective SNR for a given AWGN SNR $s$ when channel coefficients are considered as random. Then, we set the range of AWGN SNR $s$ for calibration such that $P\left(\gamma_{0.9}^{i} \leq \mathrm{SNR}_{\text {eff }}^{s} \leq \gamma_{0.001}^{i}\right) \geq$ 0.05 . Then, let $\mathrm{SNR}_{\min }$ and $\mathrm{SNR}_{\max }$ denote the minimum and maximum values of $s$, respectively. In addition, the probability $P\left(\gamma_{0.9}^{i} \leq \mathrm{SNR}_{\text {eff }}^{s} \leq \gamma_{0.001}^{i}\right)$ from the distribution of each SNR is used as a weight to calculate the total error for the optimal calibration factor.

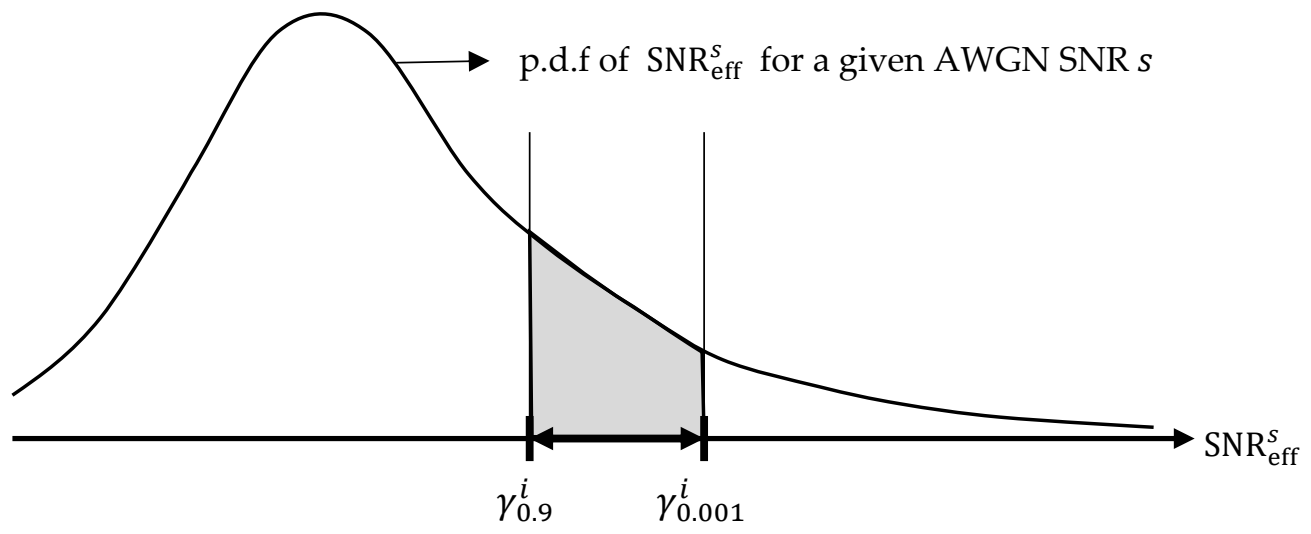

Figure 1. Selecting the additive white Gaussian noise (AWGN) signal-to-noise ratio (SNR) range from the probability density function (p.d.f) for the improved calibration procedure.

The process for deriving the optimal calibration factor is shown below in Algorithm 2.

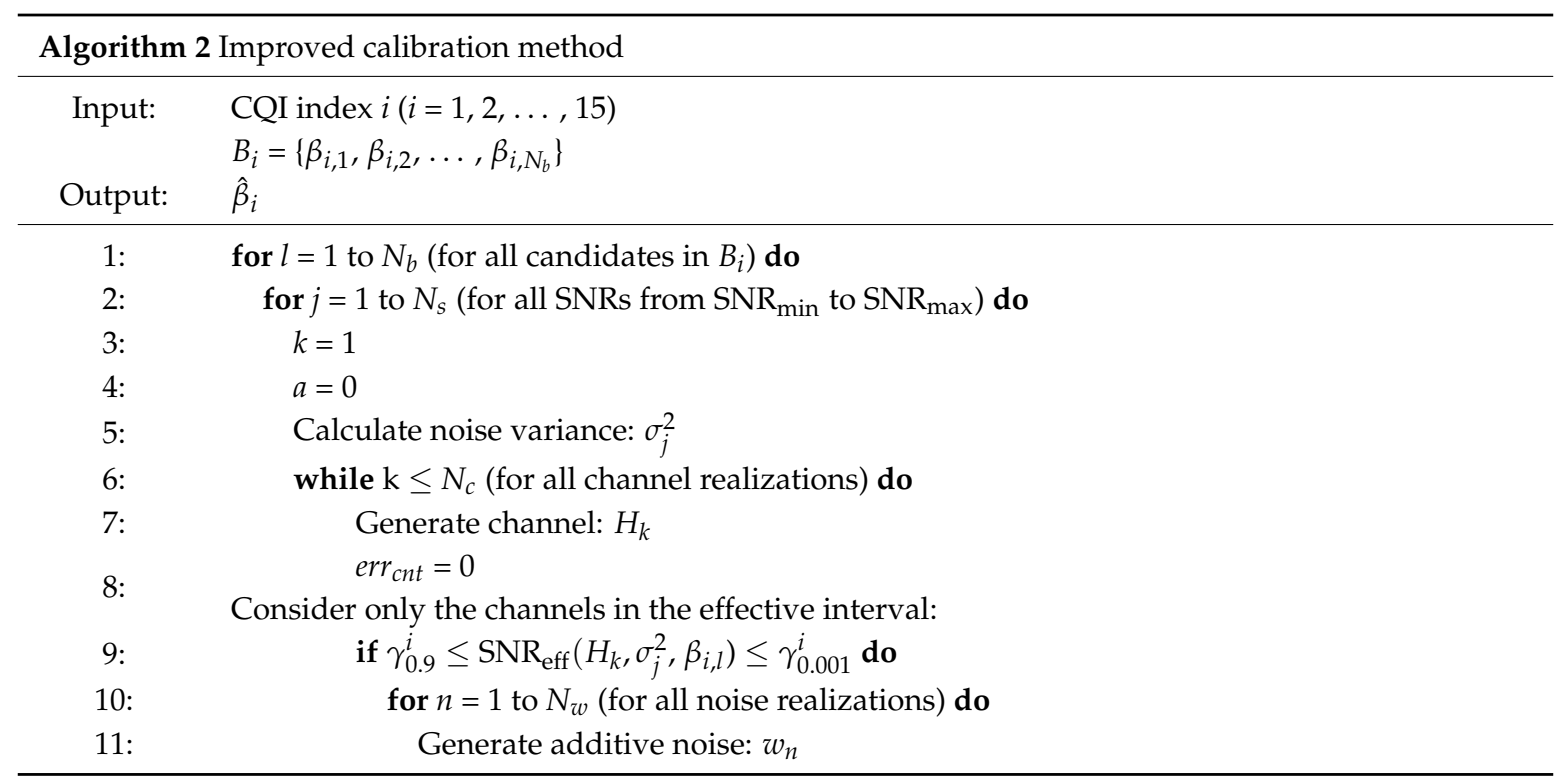




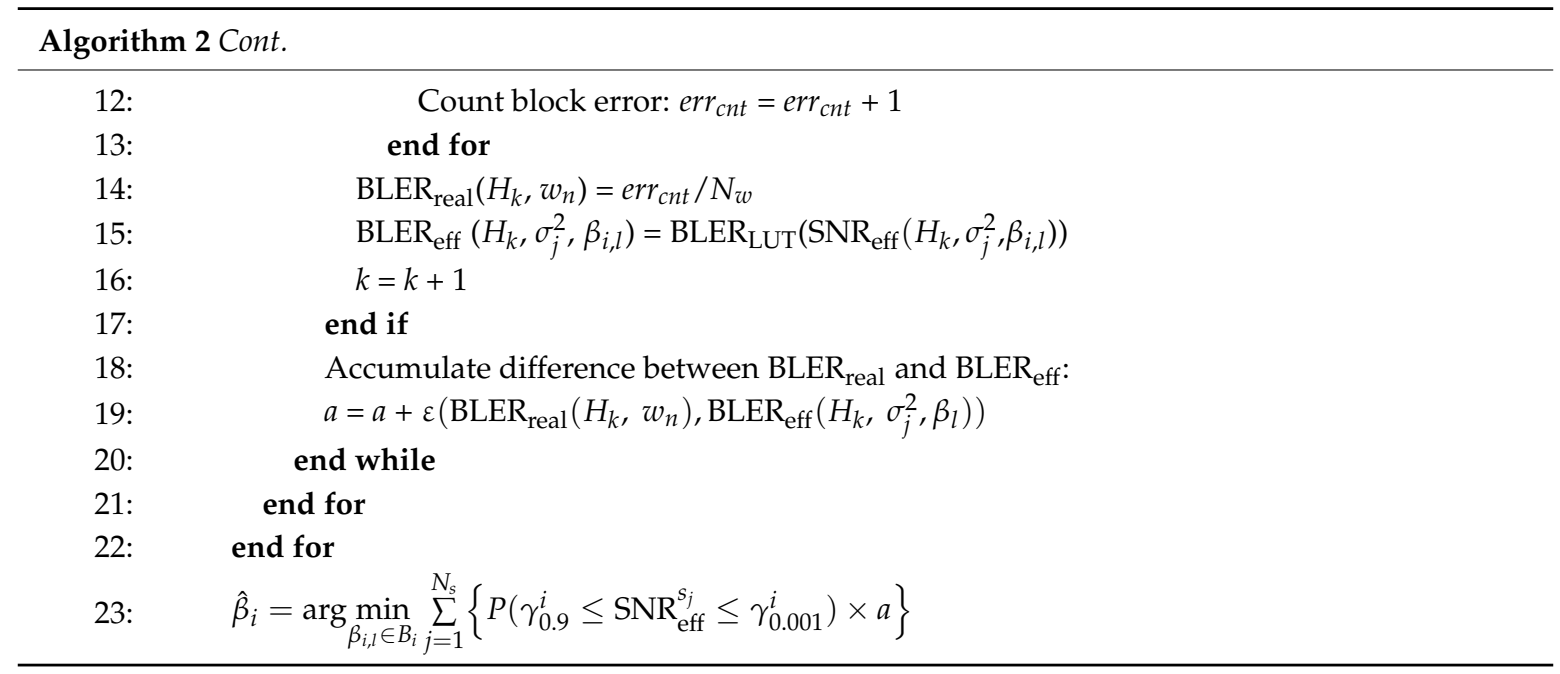

To derive the calibration factor corresponding to each CQI, $N_{b}$ candidates are specified for the calibration factor. In order to derive a calibration factor suitable for a wide range of AWGN SNR, the SNR vector of the proposed method takes the range corresponding to effective SNR ( $\left.\mathrm{SNR}_{\text {eff }}\right)$ of $5 \%$ or more obtained from the preliminary simulation, where $N_{S}$ is the total number of SNR values to simulate. The $N_{c}$ channels are generated according to the channel model. Then, noise is generated if the effective SNR is included in the effective interval for the generated channel, and the errors are counted $\left(\mathrm{err}_{\mathrm{cnt}}\right)$. The variable $\mathrm{err}_{\mathrm{cnt}}$ accumulates the number of block errors. It is used to calculate the real BLER (BLER ${ }_{\text {real }}$ ) of a physical layer for a given channel and noise. The estimated BLER $\left(B E_{\text {eff }}\right)$ is calculated using the pre-calculated $\mathrm{SNR}_{\text {eff }}$, and the error between the BLER $\mathrm{real}_{\text {ral }}$ and BLER $\mathrm{R}_{\text {eff }}$ is accumulated for all the channels. The error function of Equation (3) is again used for the error calculation. The accumulated errors are multiplied by the distribution of $\mathrm{SNR}_{\text {eff }}$ corresponding to each SNR as the weight. The $\beta_{l}$ with the smallest sum of these is determined as the optimum value of the calibration factor. The related expression is shown in Equation (4).

$$
\beta_{\text {opt }}^{\text {improv }}=\hat{\beta}_{i}=\arg \min _{\beta_{i, l} \in B_{i}} \sum_{j=1}^{N_{s}}\left\{P\left(\gamma_{0.9}^{i} \leq \mathrm{SNR}_{\text {eff }}^{s_{j}} \leq \gamma_{0.001}^{i}\right) \times \sum_{k=1}^{N_{c}} \varepsilon\left(\mathrm{BLER}_{\text {real }}, \mathrm{BLER}_{\mathrm{eff}}\right)\right\} .
$$

The complexity of a calibration method mostly depends on the number of decoding trials at the physical layer. The proposed calibration method has the exact same number of decoding trials as the average method when parameters are identically set. Specifically, the number of decoding trials for both calibration methods is $N_{b} N_{s} N_{c} N_{w}$. Therefore, it can be said that the improved calibration method does not require more computational complexity than the average method.

\section{Reduction of CQI Feedback Bits}

This section discusses schemes for reducing wideband CQI feedback bits. To date, the conventional differential CQI scheme was applied only to wideband CQI feedback. The proposed scheme also uses the difference of CQIs as the conventional method; however, the difference is not between the average CQI and each CQI, but between adjacent time channels under the assumption of the existence of time correlation in channels. The proposed scheme can be applied no matter which one is considered, i.e., wideband CQI or subband CQI. From this, the number of bits used for CQI feedback is reduced. As in LTE, we assume that CQI takes a value from 0 to 15 , and each user sends the 4-bit feedback to BS in the conventional scheme. The detailed description of the proposed scheme is shown below in Algorithm 3. 


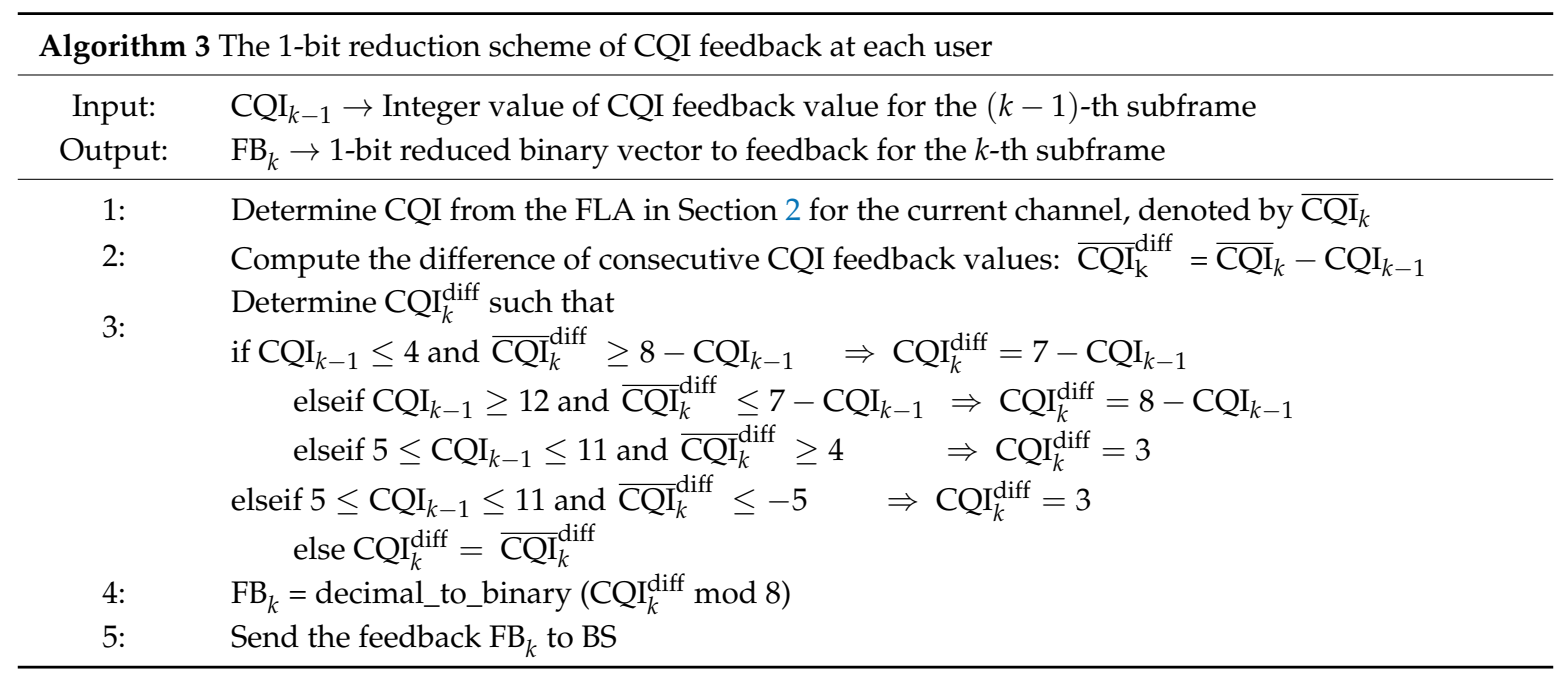

Firstly, we calculate the difference $\overline{\mathrm{CQI}}_{k}^{\text {diff }}$ between $\overline{\mathrm{CQI}}_{k}$ which is the CQI temporarily determined in a normal way for the current channel, and CQI $k_{-1}$ which is the CQI the BS used for the MCS of the previous channel. The calculated $\overline{\mathrm{CQI}}_{k}^{\text {diff }}$ is used to determine the integer value of $\mathrm{CQI}_{k}^{\text {diff }}$ which is sent to the $\mathrm{BS}$ after being converted into a binary form $\mathrm{FB}_{k}$. A detailed description of the above process is shown in Figure 2.

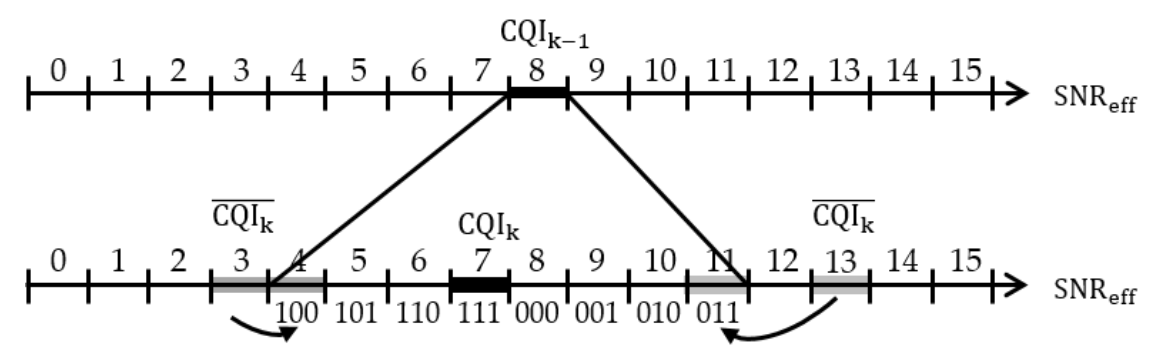

(a) $\mathrm{CQI}_{\mathrm{k}-1}=8$

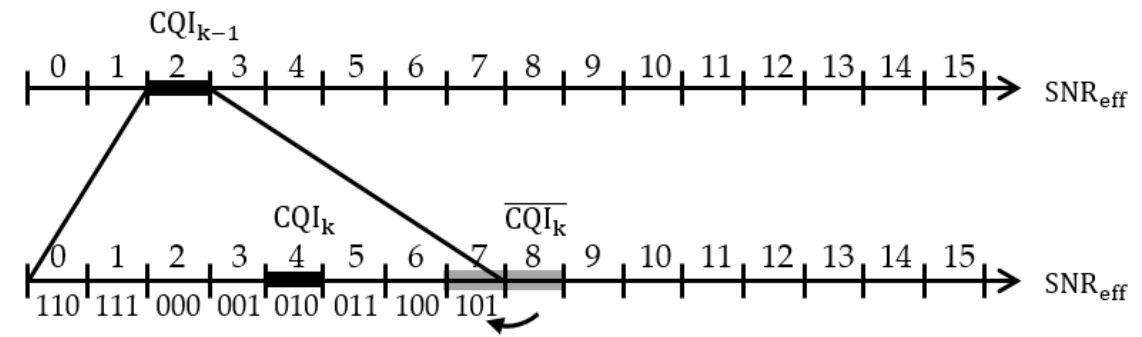

(b) $\mathrm{CQI}_{\mathrm{k}-1}=2$

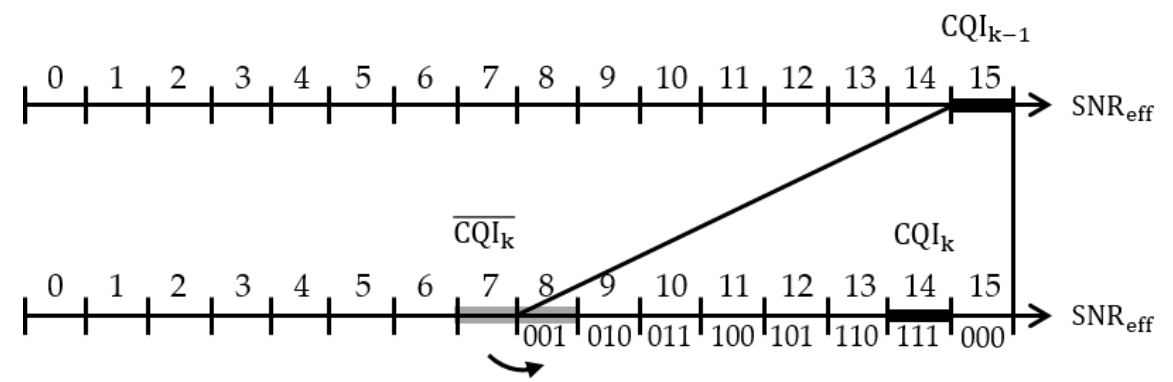

(c) $\mathrm{CQI}_{\mathrm{k}-1}=15$

Figure 2. Examples for the process of the 1-bit reduction scheme of channel quality indicator (CQI) feedback. 
The proposed scheme reduces the number of bits used for CQI feedback by 1 bit and expresses eight CQI indexes with 3 bits in total. Figure 2 shows an example of the CQI feedback range that can be represented by 3 bits according to $\mathrm{CQI}_{k-1}$. Otherwise, the $\overline{\mathrm{CQI}}_{k}$ is changed to the CQI closest to the range; then, the bit value of the CQI is fed back. In most cases, the range is specified as $\left(\mathrm{CQI}_{k-1}-4\right)$ to $\left(\mathrm{CQI}_{k-1}+3\right)$ based on $\mathrm{CQI}_{k-1}$. The above interval was determined by observing the

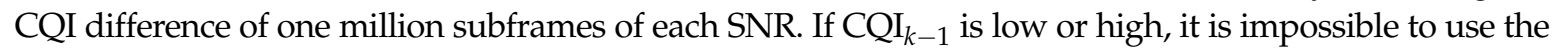
above definition of interval. Therefore, an adaptive interval setting is necessary, and the relevant examples correspond to Figure $2 b, c$.

The BS uses the $\mathrm{FB}_{k}$ fed back from the user and performs the recovery algorithm for the actual CQI, as shown in Algorithm 4. Then, it determines resource assignment, MCS, and transport block size (TBS) suitable for the current channel according to the $\mathrm{CQI}_{k}$ and transmits the data.

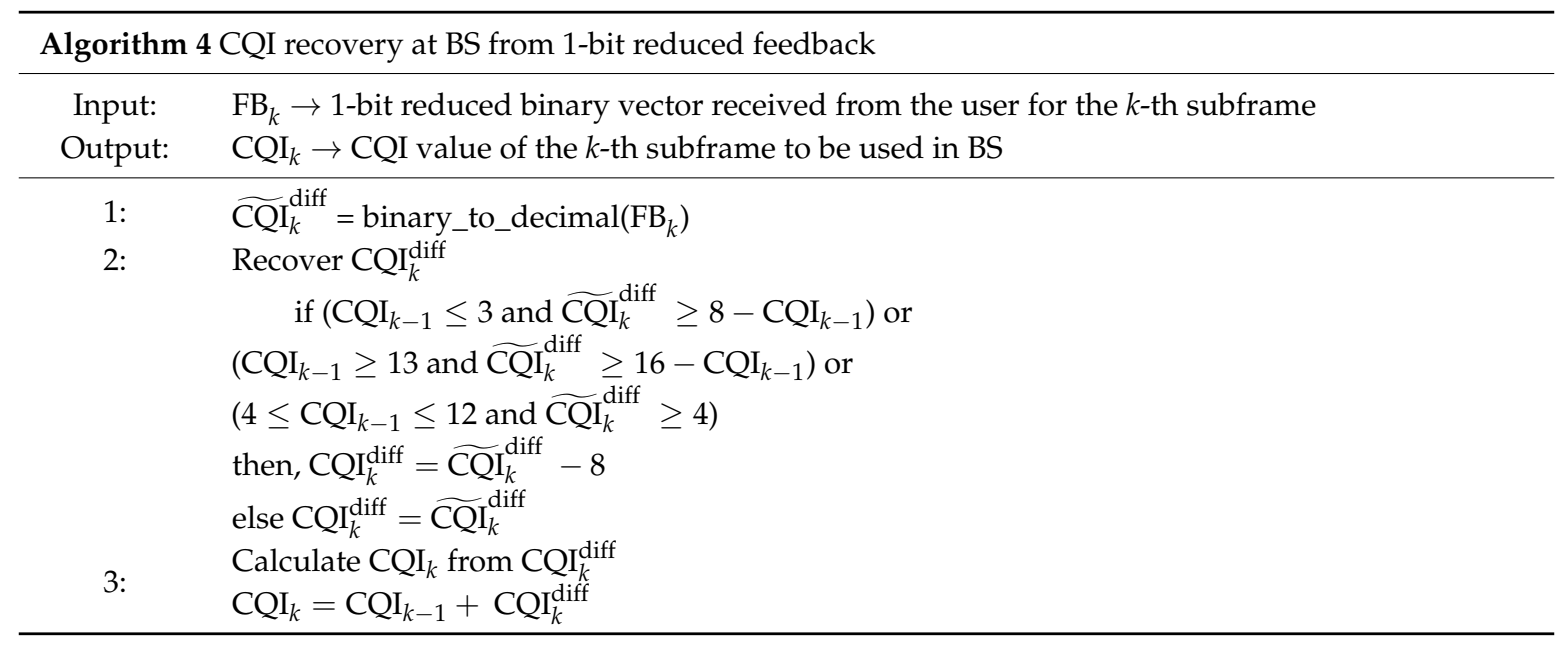

In a low-SNR environment of fading channels, we observe that the change in CQI values of adjacent channels is not large compared to high-SNR cases. In this situation, CQIs of a small level (i.e., CQI 0, CQI 1, and CQI 2) are frequently generated. We can use this observation to further reduce the bits used for CQI feedback. When the feedback CQI value for the previous channel is $\mathrm{CQI}_{k-1}=0,1,2$, we bound the CQI difference between consecutive channels as -2 to 1 , which results in 2-bit feedback for the current channel. The 2-bit CQI feedback representation method for CQIs 0, 1, and 2 is shown in Algorithm 5, and it is noted that the recovery at BS is easily described in the same way with Algorithm 4.

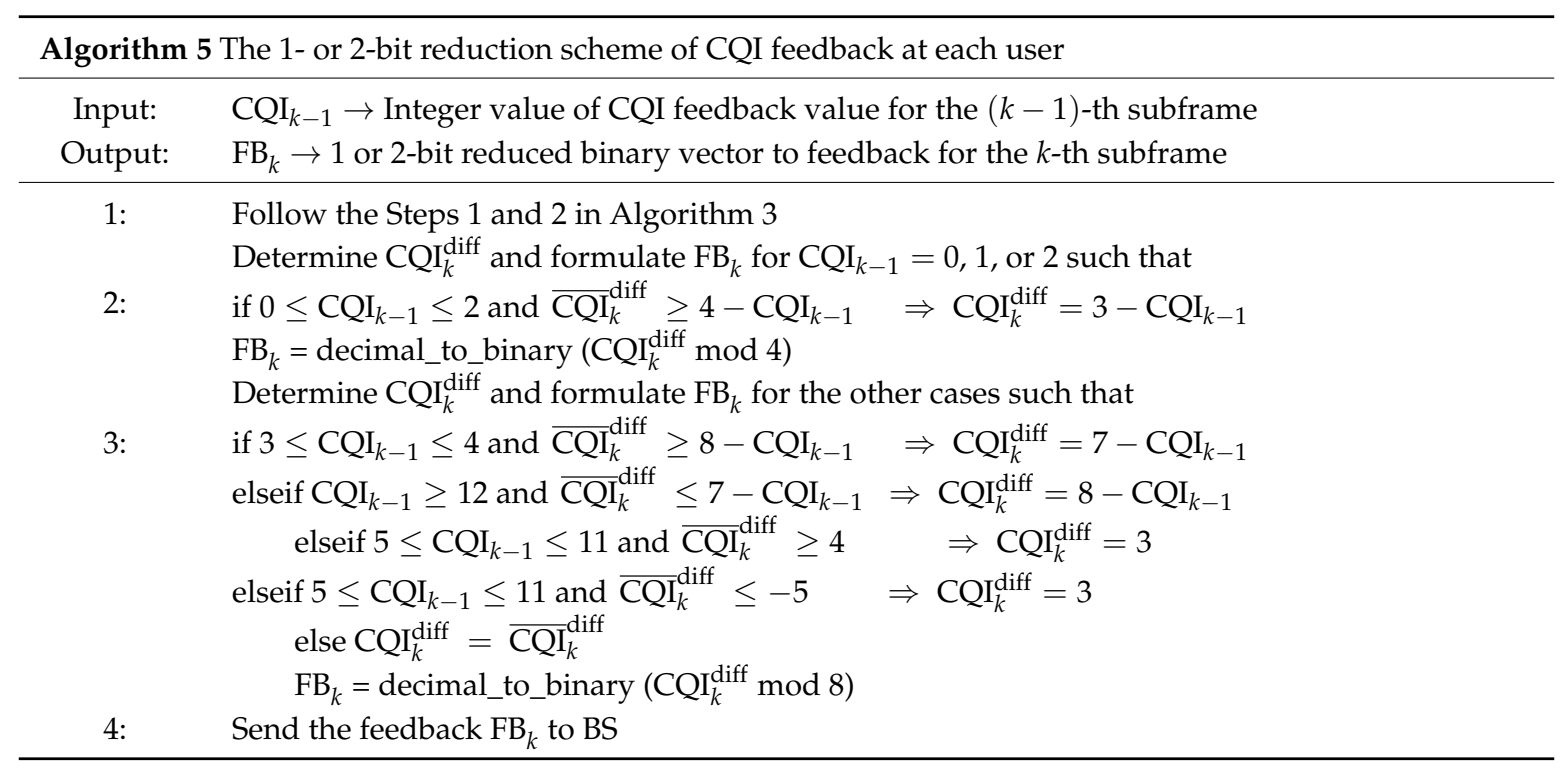


The proposed CQI feedback schemes firstly use the conventional CQI calculation. Then, they perform some comparison operations and simple substitution or subtraction based on the calculated $\mathrm{CQI}$ value. The computational complexity of the following operations is negligible compared to the first CQI calculation. Therefore, it can be said that the proposed CQI feedback schemes have almost the same computational complexity as the conventional CQI feedback scheme.

\section{Simulation Results}

In this section, we introduce the verification algorithm for evaluating the performance of the newly derived calibration factors. Also, we verify the performance of the proposed method in Sections 3 and 4 through system throughput simulations.

\subsection{Verification Algorithm for FLA}

The simulation was performed at the intermediate SNR of the interval where the $\mathrm{SNR}_{\mathrm{eff}}$ distribution ratio of each CQI was $5 \%$ or more. The information bits were encoded according to the modulation order and coding rate corresponding to $15 \mathrm{CQIs}$, and were passed through the generated channel and noise. We decoded the received data and calculated BLER $\mathrm{real} \mathrm{s}$ for all CQIs from the link-level simulation for a given channel and noise. Then, we determined a final CQI (CQI real $_{\text {) }}$ by selecting the largest CQI index such that it satisfied the condition of the target BLER among the $\mathrm{BLER}_{\text {real }}$ corresponding to each CQI. At the same time, $\mathrm{SNR}_{\text {eff }}$ corresponding to the current channel was calculated, and the effective CQI (CQI $\left.I_{\text {eff }}\right)$ was determined by calculation. If the CQI $I_{\text {real }}$ and $\mathrm{CQI}_{\text {eff }}$ were the same, the link quality was considered correct; then, verification proceeded to the next channel. Otherwise, the number of errors was increased by one before verification proceeded to the next channel.

\subsection{Performance Evaluation}

The following simulations were performed in a single-user (SU) single-input single-output (SISO) environment. It can be noted that all the proposed schemes in this paper can also be applied to MIMO systems, but the superiority of the proposed scheme over conventional ones can be sufficiently shown through just SISO simulations.

\subsubsection{Improved Calibration Method}

We present CQI feedback error rate and system throughput as measures for performance evaluation of the proposed calibration method. The common simulation parameters for evaluating the performance of the proposed calibration factors in this paper are presented in Table 1.

Table 1. Common simulation parameters for performance evaluation. SUSISO-single-user single-input single-output.

\begin{tabular}{cc}
\hline Parameter & Value \\
\hline Simulation type & SUSISO \\
Bandwidth & $1.4 \mathrm{MHz}$ \\
Carrier frequency & $2.0 \mathrm{GHz}$ \\
Subcarrier spacing & $15 \mathrm{kHz}$ \\
Cyclic prefix & Normal CP \\
Channel estimation & Perfect \\
Channel model & VehA \\
Number of PDCCH & 3 \\
Velocity & $30 \mathrm{~km} / \mathrm{h}$ \\
Receiver & Zero forcing \\
\hline
\end{tabular}




\section{- Comparison of CQI Feedback Error Rates}

In order to guarantee the reliability of the verification, 5000-10,000 channels were generated until the feedback error count exceeded 100 in each SNR. Noise occurred a maximum of 2000 times until the accumulated number of subframe transmission failures reached 100. Figure 3 shows the CQI feedback error rates for the calibration factors of the existing average calibration method and the calibration factors derived from the improved calibration method. Based on the verification algorithm in Section 5.1, the CQI feedback error rate was calculated by counting the number of times that the $\mathrm{CQI}_{\text {real }}$ determined by the BLER $\mathrm{real}_{\text {from the physical layer simulation was not equal to the CQI }}$ eff determined by $\mathrm{SNR}_{\mathrm{eff}}$.

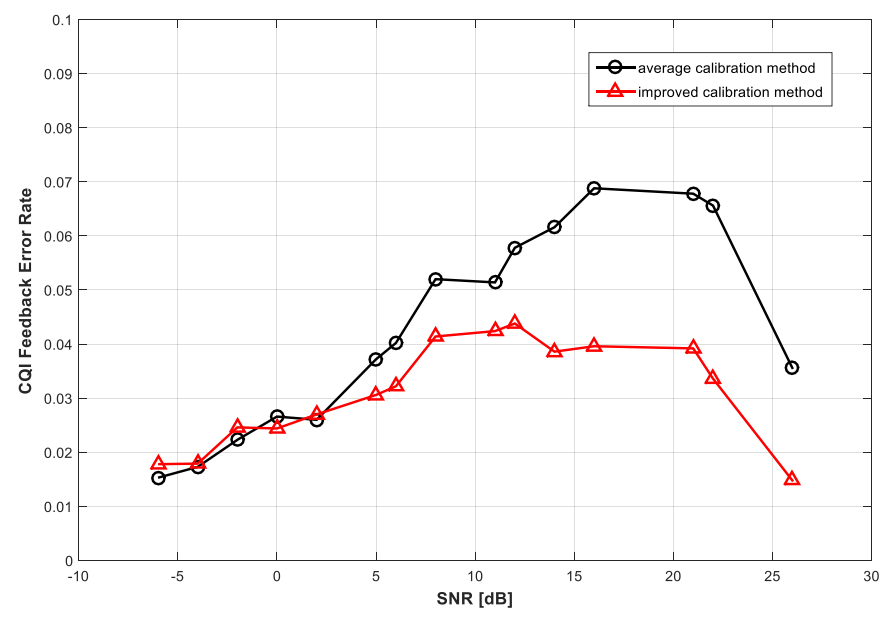

Figure 3. Comparison of CQI feedback error rates for the calibration factors derived from the average calibration method and the improved calibration method.

In the case of $\mathrm{SNR}=-2 \mathrm{~dB}$, the CQI feedback error rate of the proposed method was larger than that of the conventional method by $10.31 \%$, and for $\mathrm{SNR}=8$ and $22 \mathrm{~dB}$, the CQI feedback error rates of the proposed method were lower than those of the conventional method by $20.38 \%$ and $48.78 \%$, respectively. Based on Figure 3, the calibration factors derived from the proposed method show smaller CQI feedback error rates than the conventional method for all SNR values $>2 \mathrm{~dB}$, but they show similar to or slightly larger rates than those of the conventional method for SNR $\leq 2 \mathrm{~dB}$. This result in low-SNR environments is due to the BLER curve slope of the AWGN channel. In the proposed method, we set the SNR corresponding to BLER 0.9 and 0.001 of each CQI as the effective interval of the effective SNR, and derived the calibration factor using the more valid channel for CQI feedback; however, the calibration factor of the existing method was derived without setting it separately. For small CQI values that occur frequently in the low-SNR range, the BLER curve of the AWGN channel is relatively flat, such that the calibration factor can be calculated by sufficiently including the valid channel near the target BLER with the conventional calibration method. On the other hand, since the BLER curve of the AWGN channel is steep for a large CQI, the conventional method calculates the calibration factor without including the valid channel well. However, the proposed calibration method shows better performance than the conventional method because the effective interval works successfully.

- Comparison of System Throughputs

The system throughput was calculated by applying calibration factors derived from the average calibration method and the improved calibration method to the actual mobile communication environment. The simulation was performed over a wide SNR interval, and the system throughput for each method is shown in Table 2. 
Table 2. System throughput comparison of the improved calibration method and the average calibration methods (Mbps). SNR—signal-to-noise ratio.

\begin{tabular}{|c|c|c|c|c|c|c|c|}
\hline Calibration Method & -10 & -7 & -4 & -1 & 2 & 5 & 8 \\
\hline improved & 0.014636 & 0.056152 & 0.140616 & 0.286313 & 0.498090 & 0.783961 & 1.150098 \\
\hline average & 0.014707 & 0.056163 & 0.140429 & 0.285541 & 0.497030 & 0.782343 & 1.148416 \\
\hline Calibration Method & 11 & 14 & 17 & 20 & 23 & 26 & 29 \\
\hline $\begin{array}{l}\text { improved } \\
\text { average }\end{array}$ & $\begin{array}{l}1.592679 \\
1.590840\end{array}$ & $\begin{array}{l}2.113780 \\
2.110598\end{array}$ & $\begin{array}{l}2.675177 \\
2.666004\end{array}$ & $\begin{array}{l}3.180670 \\
3.173200\end{array}$ & $\begin{array}{l}3.551829 \\
3.545650\end{array}$ & $\begin{array}{l}3.781508 \\
3.775832\end{array}$ & $\begin{array}{l}3.904715 \\
3.900796\end{array}$ \\
\hline
\end{tabular}

The calibration factor obtained using the average method at $\mathrm{SNR} \leq-7 \mathrm{~dB}$ had a maximum system throughput of $0.07 \mathrm{kbps}$ higher than the proposed method. As described above, in an environment in which a low CQI is frequently fed back, the proposed method does not show better performance because the effective interval is not used efficiently. However, in the remaining SNR ranges, the proposed calibration method achieved high system throughput performance of at least $0.18 \mathrm{kbps}$ and up to $9.17 \mathrm{kbps}$.

\subsubsection{Reduction of CQI Feedback Bits}

Through this experiment, the system throughput of the conventional CQI feedback method and the time-coherence-based CQI feedback method were compared. The simulation set-up is listed in Table 3.

Table 3. Simulation parameters for system throughput of the proposed channel quality indicator (CQI) feedback scheme.

\begin{tabular}{cc}
\hline Parameter & Value \\
\hline Simulation type & SUSISO \\
Bandwidth & $1.4 \mathrm{MHz}$ \\
Carrier frequency & $2.0 \mathrm{GHz}$ \\
Subcarrier spacing & $15 \mathrm{kHz}$ \\
Cyclic prefix & Normal CP \\
Channel estimation & Perfect \\
Channel model & VehA \\
Velocity & $30 \mathrm{~km} / \mathrm{h}$ \\
Receiver & Zero forcing \\
Number of subframes & 100,000 \\
CQI feedback period time & $1 \mathrm{~ms}$ \\
\hline
\end{tabular}

Table 4 shows the system throughput according to the existing 4-bit wideband CQI feedback report in various SNR environments, and Table 5 shows the difference in system throughput between the existing method and the proposed method. In addition, all the system throughputs and the difference for each SNR are shown in Figure 4.

Table 4. System throughput of the 4-bit wideband CQI feedback (Mbps).

\begin{tabular}{cccccccc}
\hline SNR (dB) & $\mathbf{- 1 0}$ & $\mathbf{- 7}$ & $\mathbf{- 4}$ & $\mathbf{- 1}$ & $\mathbf{2}$ & $\mathbf{5}$ & $\mathbf{8}$ \\
\hline Throughput & 0.0146321 & 0.0560517 & 0.1404964 & 0.2860831 & 0.4981283 & 0.7843385 & 1.1504057 \\
\hline SNR (dB) & $\mathbf{1 1}$ & $\mathbf{1 4}$ & $\mathbf{1 7}$ & $\mathbf{2 0}$ & $\mathbf{2 3}$ & $\mathbf{2 6}$ & $\mathbf{2 9}$ \\
\hline Throughput & 1.5927229 & 2.1139200 & 2.6759019 & 3.1811068 & 3.5524640 & 3.7814499 & 3.9047303 \\
\hline
\end{tabular}


Table 5. System throughput differences between the existing and the proposed CQI feedback schemes (kbps).

\begin{tabular}{|c|c|c|c|c|c|c|c|}
\hline Scheme & -10 & -7 & -4 & -1 & 2 & 5 & 8 \\
\hline PROP_3bit & 0 & 0 & 0 & 0 & 0 & -0.0006399 & 0.0266000 \\
\hline PROP_2,3bit & 0 & 0.0033000 & 0.1060000 & 0.7003000 & 1.3095000 & 1.2034000 & 0.7957000 \\
\hline Scheme & 11 & 14 & 17 & 20 & 23 & 26 & 29 \\
\hline $\begin{array}{l}\text { PROP_3bit } \\
\text { PROP_2,3bit }\end{array}$ & $\begin{array}{c}-0.4101000 \\
0.1600000\end{array}$ & $\begin{array}{l}0.2572000 \\
0.2782000\end{array}$ & $\begin{array}{l}0.6247000 \\
0.6247000\end{array}$ & $\begin{array}{l}-0.3819000 \\
-0.3819000\end{array}$ & $\begin{array}{l}0.4075000 \\
0.4075000\end{array}$ & $\begin{array}{l}0.6084000 \\
0.6084000\end{array}$ & $\begin{array}{l}0.2507000 \\
0.2507000\end{array}$ \\
\hline
\end{tabular}

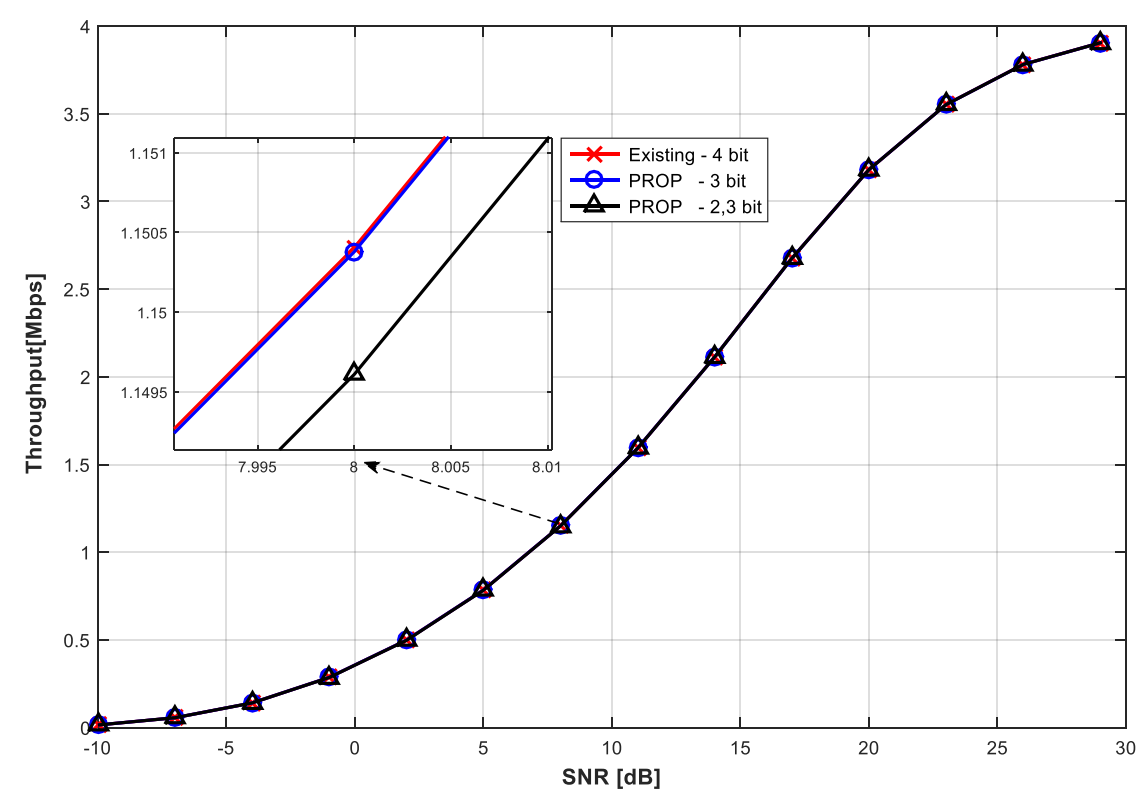

Figure 4. Comparison of system throughputs for CQI feedback schemes.

In the legend of Figure 4, "Existing" represents the conventional CQI feedback scheme in LTE, "PROP-3bit" represents the 1-bit reduced CQI feedback scheme proposed in Algorithm 3, and "PROP-2,3bit" represents the 1- or 2-bit reduced CQI feedback scheme proposed in Algorithm 5. In some SNR environments, the system throughput of existing CQI feedback schemes was slightly higher than that of the proposed scheme. However, the system throughput of the proposed technique was higher at some SNR points $(5,11$, and $20 \mathrm{~dB})$. Because of the limited range of CQI variation, the proposed method often provided more stable CQI feedback for the next channel than the conventional method. Though the proposed scheme uses limited CQI index representation for bit reduction, the above simulation results show that the system throughput difference is negligible.

Table 6 shows the average number of bits of PROP_2,3bit used for CQI feedback according to the SNR environment. From the results in Figure 4 and Tables 5 and 6, we can observe that, in low-SNR environments, only 2 bits are sufficient for CQI feedback.

Table 6. Average number of feedback bits of the 1- or 2-bit reduced CQI feedback scheme (bits).

\begin{tabular}{cccccccc}
\hline SNR (dB) & $\mathbf{- 1 0}$ & $\mathbf{- 7}$ & $\mathbf{- 4}$ & $\mathbf{- 1}$ & $\mathbf{2}$ & $\mathbf{5}$ & $\mathbf{8}$ \\
\hline Average bits used & 2.0011 & 2.0426 & 2.2593 & 2.5995 & 2.8491 & 2.9586 & 2.9922 \\
\hline SNR (dB) & $\mathbf{1 1}$ & $\mathbf{1 4}$ & $\mathbf{1 7}$ & $\mathbf{2 0}$ & $\mathbf{2 3}$ & $\mathbf{2 6}$ & $\mathbf{2 9}$ \\
\hline Average bits used & 2.9991 & 2.9999 & 3 & 3 & 3 & 3 & 3 \\
\hline
\end{tabular}




\section{Conclusions}

In this paper, we proposed an improved calibration factor derivation method for accurate link-quality determination of mobile communications in various channel environments, and we also suggested a CQI feedback bit reduction scheme for reporting downlink channel state information to BS. The improved calibration method sets the effective interval for the effective SNR to derive the optimal calibration factor used by the MIESM using more valid channels in a wide SNR range. The CQI feedback bit reduction scheme applies a differential CQI technique based on the time correlation between adjacent channels, which change over time, and decreases the number of bits used for CQI feedback.

The two proposed methods were evaluated and analyzed through simulation. Firstly, the calibration factor derived from the improved calibration method reduced the CQI feedback error rate by $48.8 \%$ over the existing calibration factor in the range of SNR $>2 \mathrm{~dB}$. Also, the system throughput of proposed scheme was a maximum of $9.17 \mathrm{~dB}$ higher than that of the conventional scheme at SNR $\geq-7 \mathrm{~dB}$. This means that, by setting the effective interval in the wide SNR range, the newly derived calibration factor performs better than the existing one. Next, using a reduction of the CQI feedback scheme can be used to transmit uplink scheduling information by a reduced number of bits, though the system throughput is almost the same or a maximum of $1.3 \mathrm{kbps}$ lower than the conventional single 4-bit wideband CQI feedback.

Author Contributions: Conceptualization, H.P. and H.L.; methodology, H.L. and H.P.; software, H.L. and H.K.; validation, H.L., H.P. and H.K.; formal analysis, H.L., H.P. and H.K.; investigation, H.L. and H.K.; resources, H.L.; data curation, H.P. and H.L.; writing—original draft preparation, H.L., H.P. and H.K.; writing-review and editing, H.L. and H.P.; visualization, H.L. and H.K.; supervision, H.P.; project administration, H.P.

Funding: This research was supported by the Basic Research Program through the National Research Foundation of Korea (NRF) funded by the Ministry of Education (NRF-2018R1D1A1B07051108).

Conflicts of Interest: The authors declare no conflict of interest.

\section{References}

1. Ku, G.; Walsh, J.M. Resource allocation and link adaptation in LTE and LTE advanced: A tutorial. IEEE Commun. Surv. Tutor. 2015, 17, 1605-1633. [CrossRef]

2. Love, R.; Bachu, R.; Classon, B.; Nory, R.; Stewart, K.; Sun, Y. Channel Quality Indicator for Time, Frequency and Spatial Channel in Terrestrial Radio Access Network. U.S. Patent 9184898, 10 November 2015.

3. Goldsmith, A.J.; Chua, S. Adaptive coded modulation for fading channels. IEEE Trans. Commun. 1998, 46, 595-602. [CrossRef]

4. Fantacci, R.; Marabissi, D.; Tarchi, D. Adaptive modulation and coding techniques for OFDMA systems. IEEE Trans. Wirel. Commun. 2009, 8, 4876-4883. [CrossRef]

5. Kastrinogiannis, T.; Tsiropoulou, E.-E.; Papavassiliou, S. Utility-based uplink power control in CDMA wireless networks with real-time services. In Proceedings of the International Conference on Ad-Hoc Networks and Wireless, Sophia Antipolis, France, 10-13 September 2008; pp. 307-320.

6. Kastrinogiannis, T.; Tsiropoulou, E.-E.; Papavassiliou, S. Uplink power control in QoS-aware multi-service CDMA wireless networks. J. Commun. 2009, 4, 654-668.

7. Rudolf, M.; Dick, S.G.; Miller, J.M. Reliability Detection of Channel Quality Indicator (CQI) and Application to Outer Loop Power Control. U.S. Patent 8204450, 19 June 2012.

8. Ramiro-Moreno, J.; Pedersen, K.I.; Mogensen, P.E. Network performance of transmit and receive antenna diversity in HSDPA under different packet scheduling strategies. In Proceedings of the IEEE Vehicular Technology Conference, Vancouver, BC, Canada, 24-28 September 2002; pp. 482-486.

9. Pedersen, K.I.; Mogensen, P.E. Performance of WCDMA HSPDA in a beamforming environment under code constraints. In Proceedings of the IEEE Vehicular Technology Conference, Orlando, FL, USA, 6-9 October 2003; pp. 995-999. 
10. Peppas, K.; Alexiou, A.; Lazarakis, F.; Al-Gizawi, T.; Axiotis, D.I. Performance evaluation at the system level of reconfigurable space-time coding techniques for HSDPA. EURASIP J. Appl. Signal Process. 2005, 1656-1667. [CrossRef]

11. Tuomaala, E.; Wang, H. Effective SINR approach of link to system mapping in OFDM/multi-carrier mobile network. In Proceedings of the 2nd Asia Pacific Conference on Mobile Technology, Applications and Systems, Guangzhou, China, 15-17 November 2005; pp. 1-5.

12. Brueninghaus, K.; Astely, D.; Salzer, T.; Visuri, S.; Alexiou, A.; Karger, S.; Seraji, G.A. Link performance models for system level simulations of broadband radio access systems. In Proceedings of the IEEE International Symposium on Personal, Indoor and Mobile Radio Communications, Berlin, Germany, 11-14 September 2005; pp. 2306-2311.

13. Cipriano, A.M.; Visoz, R.; Salzer, T. Calibration issues of PHY layer abstractions for wireless broadband systems. In Proceedings of the IEEE Vehicular Technology Conference, Calgary, BC, Canada, 21-24 September 2008; pp. 1-5.

14. Yang, J.; Zhao, H.; Wang, W.; Zhang, C. An effective SINR mapping models for 256QAM in LTE-Advanced system. In Proceedings of the IEEE 25th Annual International Symposium on Personal, Indoor, and Mobile Radio Communication (PIMRC), Washington, DC, USA, 2-5 September 2014; pp. 343-347.

15. Donthi, S.N.; Mehta, N.B. An accurate model for EESM and its application to analysis of CQI feedback schemes and scheduling in LTE. IEEE Trans. Wirel. Commun. 2011, 10, 3436-3448. [CrossRef]

16. Freudenthaler, K.; Springer, A.; Wehinger, J. Novel SINR-to-CQI mapping maximizing the throughput in HSDPA. In Proceedings of the IEEE Wireless Communications and Networking Conference, Kowloon, China, 11-15 March 2007; pp. 2233-2237.

17. Vien, Q.-T.; Nguyen, H.X. Network coding-based channel quality indicator reporting for two-way multi-relay networks. Wirel. Commun. Mob. Comput. 2014, 14, 1471-1483. [CrossRef]

18. 3GPP Spec. Evolved Universal Terrestrial Radio Access (E-UTRA), Physical Layer Procedure, TS 36.213, v.13.0.0. May 2016. Available online: https:/ / www.etsi.org/deliver/etsi_ts/136200_136299/136213/13.00. 00_60/ts_136213v130000p.pdf (accessed on 13 November 2018).

19. Murakami, T.; Miyazaki, M.; Ishida, S.; Fukuda, A. Wireless-LAN based CSI monitoring system for object detection. Electronics 2018, 7, 290. [CrossRef]

20. Jensen, T.L.; Kant, S.; Wehihger, J.; Fleury, B.H. Fast link adaptation for MIMO OFDM. IEEE Trans. Veh. Technol. 2010, 59, 3766-3778. [CrossRef]

21. Hanzaz, Z.; Schotten, H.D. Analysis of effective SINR mapping models for MIMO OFDM in LTE Systems. In Proceedings of the International Wireless Communications and Mobile Computing Conference, Sardinia, Italy, 1-5 July 2013; pp. 1509-1515. 